\title{
Toxicity of chlorine to zebrafish embryos
}

\author{
Michael L. Kent ${ }^{1, *}$, Cari Buchner ${ }^{2}$, Carrie Barton ${ }^{2}$, Robert L. Tanguay ${ }^{2}$ \\ ${ }^{1}$ Department of Microbiology, and ${ }^{2}$ Department of Environmental and Molecular Toxicology, Oregon State University, \\ Corvallis, Oregon, USA
}

\begin{abstract}
Surface disinfection of fertilized fish eggs is widely used in aquaculture to reduce extraovum pathogens that may be released from brood fish during spawning, and this is routinely used in zebrafish Danio rerio research laboratories. Most laboratories use approximately 25 to $50 \mathrm{ppm}$ unbuffered chlorine solution for 5 to $10 \mathrm{~min}$. Treatment of embryos with chlorine has significant germicidal effects for many Gram-negative bacteria, viruses, and trophozoite stages of protozoa, but is less effective against cyst or spore stages of protozoa and certain Mycobacterium spp. Therefore, we evaluated the toxicity of unbuffered and buffered chlorine solutions to embryos exposed at 6 or $24 \mathrm{~h}$ post-fertilization (hpf) to determine whether higher concentrations can be used for treating zebrafish embryos. Most of our experiments entailed using an outbred line (5D), with both mortality and malformations as endpoints. We found that $6 \mathrm{hpf}$ embryos consistently were more resistant than $24 \mathrm{hpf}$ embryos to the toxic effects of chlorine. Chlorine is more toxic and germicidal at lower $\mathrm{pH}$, and chlorine causes elevated $\mathrm{pH}$. Consistent with this, we found that unbuffered chlorine solutions ( $\mathrm{pH}$ ca. 8-9) were less toxic at corresponding concentrations than solutions buffered to $\mathrm{pH} 7$. Based on our findings here, we recommend treating $6 \mathrm{hpf}$ embryos for $10 \mathrm{~min}$ and $24 \mathrm{hpf}$ embryos for 5 min with unbuffered chlorine solution at $100 \mathrm{ppm}$.
\end{abstract}

KEY WORDS: Danio rerio $\cdot$ Zebrafish $\cdot$ Chlorine $\cdot$ Mortality $\cdot$ Malformations

\section{INTRODUCTION}

Surface disinfection of fertilized fish eggs has been widely used in aquaculture to reduce or eliminate extraovum pathogens that may be released from brood fish during spawning. This is an integral step used by aquaculturists in attempt to avoid maternal transmission. Iodine is the disinfectant of choice for salmonids and other species, whereas chlorine is most often used with zebrafish Danio rerio. Most zebrafish research laboratories disinfect the embryos with chlorine with new introductions to a facility, and some use this procedure on a routine basis with each spawn. Common concentrations and doses used by most zebrafish laboratories are between 25 and $50 \mathrm{ppm}$ for about $10 \mathrm{~min}$ (Westerfield 2007, Harper \& Lawrence 2011). These regimens were established without extensive evaluation of the lethality and toxicity of chlorine. The germicidal properties of chlorine are reduced at higher $\mathrm{pH}$ levels, and addition of chlorine to water raises the $\mathrm{pH}$ of water (Clark et al. 1989). These standard protocols used in zebrafish laboratories do not include buffering the chlorine solutions.

The treatment regimens that are presently used should be effective at significantly reducing the viability of most Gram-negative bacteria (Goñi-Urriza et al. 2000) and trophozoite stages of protozoa (Vaerewijck et al. 2012). However, these concentrations are less effective for protozoan spores, cysts, and helminth eggs. For example, Pseudoloma neurophilia (Microsporidia) is the most common infectious agent seen in zebrafish colonies, and Ferguson et al. (2007) showed that 25 and $50 \mathrm{ppm}$ of chlorine in which the solution is not $\mathrm{pH}$ adjusted (as used by most laboratories) killed only 40 and $60 \%$ of the spores of this microsporidium, respectively. Numerous studies have evaluated the toxicity of chlorine to fish embryos and larvae (e.g. Morgan \& Prince 1997). These studies involved longer exposure times (e.g. 
24 to $48 \mathrm{~h}$ ) and reported lethal concentrations ( $\left.\mathrm{LC}_{50}\right)$ considerably lower than 25 to $50 \mathrm{ppm}$.

Here we evaluated the toxicity of chlorine to zebrafish embryos exposed to chlorine solution at levels and durations routinely used by zebrafish researchers. We also conducted experiments in which the solutions were adjusted to $\mathrm{pH} 7$ or not adjusted, and used mortality and malformations in the fish as endpoints.

\section{MATERIALS AND METHODS}

\section{Fish}

All exposure studies were conducted at the Sinnhuber Aquatic Research Laboratory (SARL), Oregon State University, Corvallis, OR, USA, using an outbred wild type zebrafish line referred to as 5D. Embryos were obtained from large group spawns from $\sim 90 \mathrm{~cm}$ circular tanks holding 800 to 1000 adult fish, in which eggs are collected with a catch system.

\section{Exposure}

Thirty-two eggs per treatment (2 replicates of 16) were exposed for each concentration of chlorine (J. T. Baker Analytical grade 5.8\%), in which the solution was either buffered to $\mathrm{pH} 7$ with acetic acid or was left unbuffered. The pH was recorded with an American Marine Pinpoint pH Monitor. The chlorine solutions were prepared within $1 \mathrm{~h}$ of exposure, and the water source was from the reverse osmosis system at the SARL (calcium hardness 17 ppm, total alkalinity $28 \mathrm{ppm})$.

Embryos from the same clutch were exposed at either 6 or $24 \mathrm{~h}$ post-fertilization (hpf) for either 5 or $10 \mathrm{~min}$. These 2 ages of embryos were chosen because embryos are usually treated within a few hours of fertilization, but sometimes treatments occur following shipment of embryos. Exposure was accomplished by first placing eggs in $50 \mathrm{ml}$ plastic conical tubes in which the bottom had been replaced with $500 \mu \mathrm{m}$ screen. Containers were then transferred to the appropriate solution, held for either 5 or $10 \mathrm{~min}$, and then rinsed with chlorine-free water. In the first trial, fish were exposed to buffered ( $\mathrm{pH} \mathrm{7)}$ or unbuffered chlorine solutions ranging from 0 to $100 \mathrm{ppm}$ at the following concentrations and $\mathrm{pH}$ values (for unbuffered solutions): 0 ppm (pH 5), $6.25 \mathrm{pm}(\mathrm{pH}$ 5), 12.5 ppm (pH 5.9), 25 ppm (pH 6.7), 50 ppm (pH 7.5), 100 ppm (pH 8.3).
Following the first experiment described above, we conducted a second trial with higher concentrations of chlorine, exposing embryos at $6 \mathrm{hpf}$ for $5 \mathrm{~min}$ to the following concentrations of chlorine in unbuffered solutions: 0 ppm (pH 5.7), 100 ppm (pH 8.5), 125 ppm (pH 9.0), 150 ppm (pH 9.4), 175 ppm (pH 9.5), or $200 \mathrm{ppm}$ (pH 9.8). A total of 64 embryos (4 plates at 16 embryos plate ${ }^{-1}$ ) were exposed at each concentration. As there was high survival at 200 ppm, a third experiment using 225 ppm (pH 9.5) and 250 ppm (pH 9.7) unbuffered chlorine solution was conducted, with 64 embryos per concentration (2 replicates of 32).

A larger-scale exposure study using unbuffered chlorine was then conducted using 2 selected exposure regimes based on findings of the trials above. A total of 400 embryos at $6 \mathrm{hpf}$ were exposed for $5 \mathrm{~min}$ at $175 \mathrm{ppm}(\mathrm{pH} 8.9)$, and the same numbers of $24 \mathrm{hpf}$ embryos of both lines were exposed at $125(\mathrm{pH} 8.4)$ ppm for $5 \mathrm{~min}$. Embryos were exposed by placing them in $400 \mathrm{ml}$ tri-pours with mesh bottoms as described above, rinsed following exposure, divided into 4 groups of 100 embryos, and incubated in $150 \mathrm{~mm}$ Petri dishes with $150 \mathrm{ml}$ water.

\section{Toxicity evaluation}

Following exposure, embryos were placed individually in $100 \mu \mathrm{l}$ E2 embryo medium (Westerfield 2007) solution in $340 \mu \mathrm{l}$ wells (96 wells plate ${ }^{-1}$; BD Biosciences). This medium had total alkalinity of $76 \mathrm{ppm}$ and calcium hardness of 29 ppm.

With 6 hpf exposures, embryos were evaluated at $24 \mathrm{hpf}$ for mortality, developmental delay, spontaneous movement, and notochord malformations. For $24 \mathrm{hpf}$ exposures, embryos were evaluated $30 \mathrm{~min}$ post exposure for immediate mortality. The survivors from both time period exposures were also evaluated at $5 \mathrm{dpf}$ for the following endpoints: mortality, natural hatch, any aberrations of the yolk sac, axis, eye, snout, jaw, otolith, pericardial edema, brain, somites, pectoral fin, caudal fin, pigmentation, circulatory system, trunk, swim bladder, notochord, and touch response (head and tail) following protocols routinely used in the Tanguay laboratory for zebrafish embryo toxicity assays (Truong et al. 2011, Kim \& Tanguay 2013). Death was determined by lack of heartbeat.

\section{Statistics}

Statistical differences between each exposure concentration were compared to controls (0 ppm chlo- 
rine) in each trial using SigmaPlot 11 (Systat Software). Fisher's exact test was used for groups of 32 or less, as a separate control was used with each exposure group. Experiments with greater numbers (e.g. $\mathrm{n}=64$ ) were evaluated with a chi-squared test, as the SigmaPlot program automatically decides which test it is qualified to execute, and the former test was rejected as appropriate for the larger data sets. Differences between a given concentration and $0 \mathrm{ppm}$ were considered statistically different at $\mathrm{p}<0.05$.

\section{RESULTS}

The various trials consistently showed correlations with higher incidence of mortality and malformations associated with higher chlorine concentrations. Overall, mortality and malformations in the 3 experiments

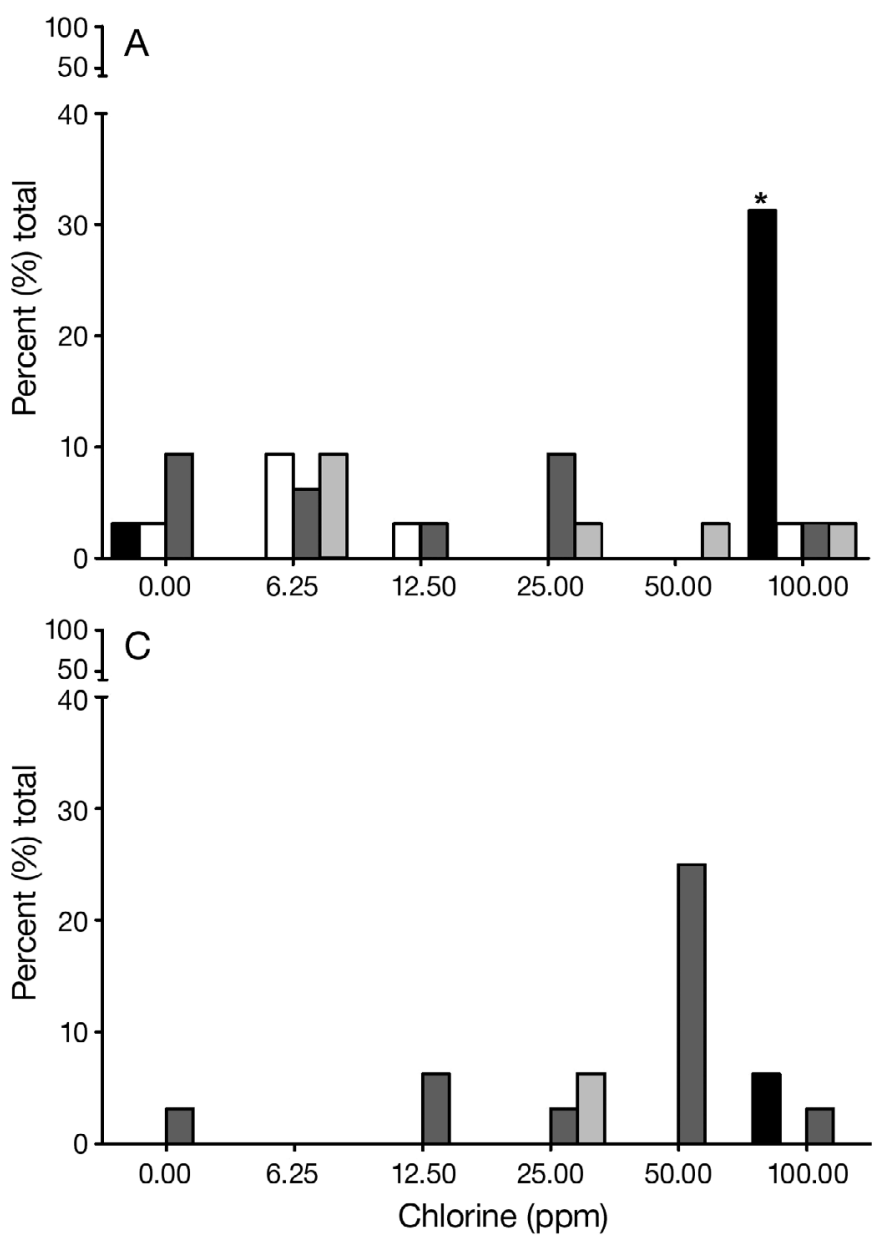

were statistically greater than controls only at levels $>100$ ppm (Figs. 1 \& 2). Solutions with chlorine adjusted to a pH 7 were consistently more toxic than unbuffered solutions, the latter of which ranged up to over pH 9 at the higher concentrations. The $24 \mathrm{hpf}$ embryos were more susceptible to chlorine exposure than 6 hpf embryos, and exposure for $10 \mathrm{~min}$ was associated with greater mortality than 5 min exposure (Figs. 1 \& 2).

Regarding $6 \mathrm{hpf}$ embryos, in the first trial (Fig. 1A,B), at 5 and $10 \mathrm{~min}$, we observed no statistically significant sublethal or lethal effects above background ( $0 \mathrm{ppm}$ ) for either solution (buffered or unbuffered) at $50 \mathrm{ppm}$ or below. At $100 \mathrm{ppm}$, high mortality occurred at both exposure time points with chlorine at $\mathrm{pH}$. With unbuffered chlorine at $100 \mathrm{ppm}$ for $10 \mathrm{~min}$, the $6 \mathrm{hpf}$ fish showed $40 \%$ mortality (Fig. 1B). About $15 \%$ of the surviving
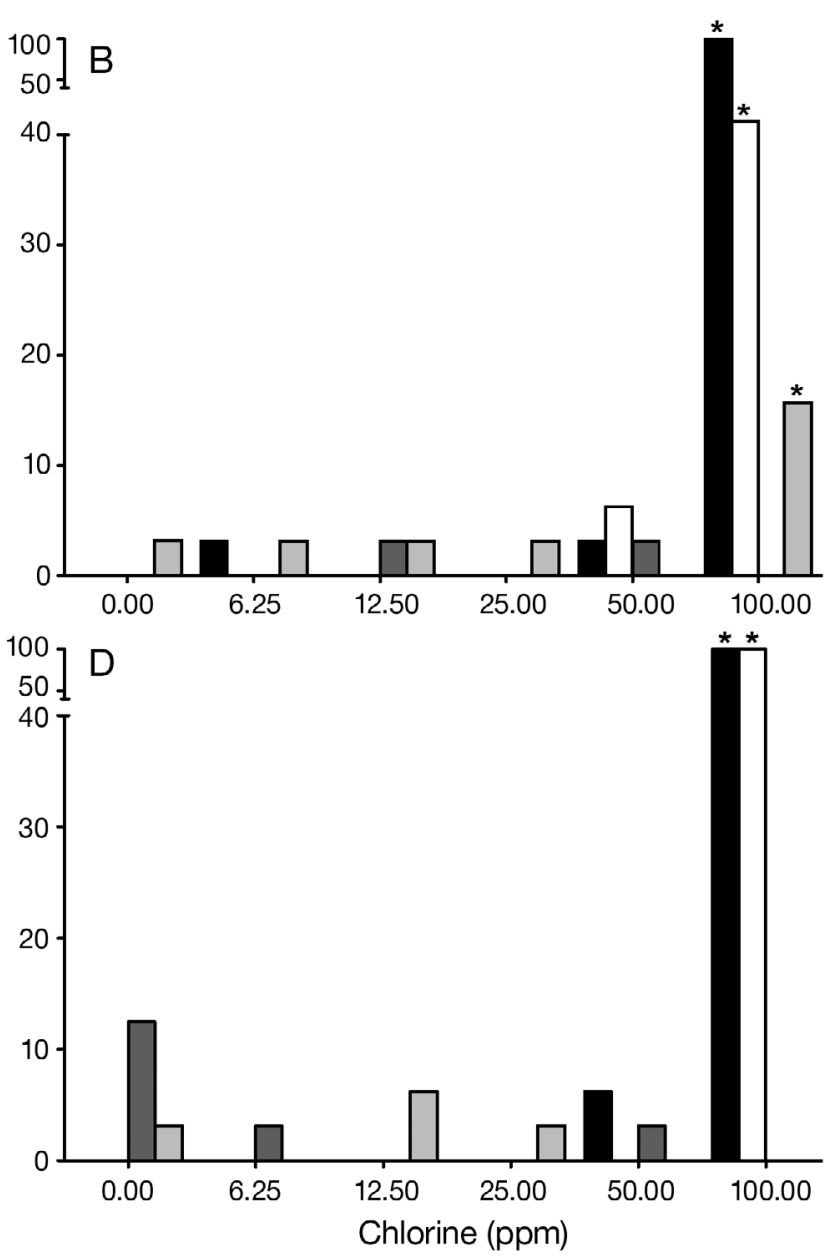

Fig. 1. Danio rerio. Mortality and malformations in 6 and $24 \mathrm{~h}$ post fertilization (hpf) embryos exposed to chlorine solution buffered to pH 7 or unbuffered solution: (A) 6 hpf, 5 min exposure, (B) 6 hpf, 10 min exposure, (C) 24 hpf, 5 min exposure, (D) $24 \mathrm{hpf}, 10 \mathrm{~min}$ exposure. In all panels, black and white bars indicate mortality rates in buffered and unbuffered solutions, respectively. Dark and light grey bars indicate malformation rates in buffered and unbuffered solutions, respectively. Asterisks indicate significant differences from 0 ppm (Fisher's exact test, $\mathrm{p}<0.05$ ) 


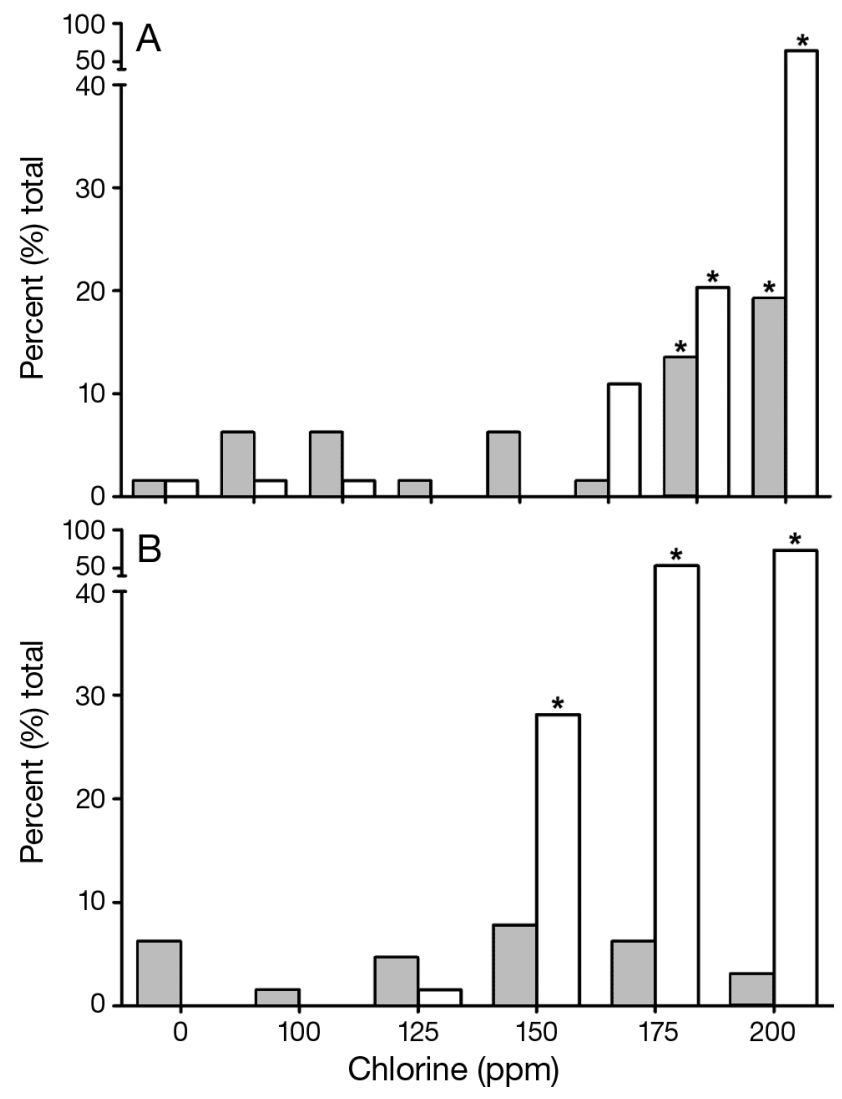

Fig. 2. Danio rerio. Rates of mortality (white bars) and malformations (grey bars) in (A) $6 \mathrm{~h}$ post fertilization (hpf) and (B) $24 \mathrm{hpf}$ embryos exposed to high-concentration unbuffered chlorine solution for $5 \mathrm{~min}$. Asterisks indicate significant differences from 0 ppm (chi-squared test, $\mathrm{p}<0.05$ )

embryos exposed to $100 \mathrm{ppm}$ unbuffered chlorine for 10 min exhibited malformations, including yolk sac edema, pericardial edema, body axis curvature, and snout and jaw malformations.

The $24 \mathrm{hpf}$ exposed embryos showed a similar pattern, except no significant mortality was observed with a 5 min exposure, including $100 \mathrm{ppm}$. However, significant $(\mathrm{p}<0.05)$ malformations were observed at $50 \mathrm{ppm}$ (Fig. 1) in buffered chlorine. All embryos died when exposed for $10 \mathrm{~min}$ at $100 \mathrm{ppm}$ chlorine, with both unbuffered and buffered solutions.

The second experiment used higher concentrations of only unbuffered chlorine at $5 \mathrm{~min}$ (Fig. 2). The $6 \mathrm{hpf}$ embryos showed minimal mortality and malformations (Fig. 2). In contrast, $24 \mathrm{hpf}$ embryos showed significantly increased malformations at $150 \mathrm{ppm}$ and above, but mortality was still less than $30 \%$ at these high levels. Exposure of $6 \mathrm{hpf}$ embryos to 200, 225 , or $250 \mathrm{ppm}$ unbuffered chlorine solution in another trial resulted in mortalities and malformations above $10 \%$ only at the highest concentration.
A large-scale exposure, using several embryos exposed to unbuffered chlorine at 6 and $24 \mathrm{hpf}$ again showed that the embryos could tolerate high levels of unbuffered chlorine. The $6 \mathrm{hpf}$ embryos exposed to $175 \mathrm{ppm}$ chlorine showed no mortality and $2.0 \%$ malformations, compared to 0.5 and $2.0 \%$ in the controls, respectively. The $24 \mathrm{hpf}$ embryos exposed to $125 \mathrm{ppm}$ showed $3.0 \%$ mortality and $1.8 \%$ malformations, compared to 1.0 and $3.0 \%$ in the controls, respectively. We found no statistical differences between the exposure concentrations in controls with both exposure regimens.

\section{DISCUSSION}

Our study demonstrated that zebrafish embryos, particularly those at $6 \mathrm{hpf}$, can tolerate higher chlorine concentrations than the routine protocol of 25 or $50 \mathrm{ppm}$ for 10 min that is used by most zebrafish laboratories (Westerfield 2007, Harper \& Lawrence 2011). Buffering of the chlorine solutions to $\mathrm{pH} 7$ was consistently associated with higher corresponding levels of mortality and malformations. Addition of chlorine to water increases $\mathrm{pH}$, and as $\mathrm{pH}$ increases significantly above $\mathrm{pH} 7$, the germicidal properties and toxic activity of chlorine are reduced (Clark et al. 1989). For example, using the formula developed by Clark et al. (1989), adjustment of $\mathrm{pH}$ from 9 to 7 should double the toxicity to microorganisms (based on results with Giardia; Health Canada 2004). The most germicidal form of chlorine is $\mathrm{HOCl}$, and above $\mathrm{pH} 7.5$ very little chlorine exists in this form, while most becomes the less active form, $-\mathrm{OCl}$ (Clark et al. 1989). In a previous study with Pseudoloma neurophilia (Ferguson et al. 2007), 100 ppm-buffered chlorine ( $\mathrm{pH} 7$ ) killed almost $100 \%$ of the spores, while the unbuffered chlorine ( $\mathrm{pH}$ 9.5) killed about $80 \%$.

Embryos at 6 hpf usually tolerated chlorine better than 24 hpf embryos exposed under similar conditions. Therefore, the general recommendation would be to conduct chlorine treatments at close to $6 \mathrm{hpf}$. This is not always possible, as often laboratories treat embryos after receiving them from other facilities. In this case, the duration or concentration of chlorine should be reduced. Based on our findings here, our present general recommendation is to treat embryos for $5 \mathrm{~min}$ with unbuffered chlorine at $100 \mathrm{ppm}$ to obtain minimal mortalities and malformations. Researchers may also consider using higher concentrations or time exposures when importing new fish for the first time into the facility. This would likely result 
in a higher level of toxic changes with the first generation, but would provide better protection for introduction of exotic pathogens to a facility. For example, our data indicate that exposing $6 \mathrm{hpf}$ embryos for 10 min in unbuffered chlorine solution should result in mortalities and malformations well below $50 \%$. Once a line is established, low-concentration treatments could be employed, as the goal in this situation is mostly pathogen reduction, rather than complete avoidance within a facility. Conversely, one might consider not treating embryos with chlorine at all for specific toxicology or behavioral experiments, as we still have not elucidated all of the potential long-term effects of these chlorine treatments. The SARL's rapid throughput testing facility indeed does not bleach embryos prior to testing, largely because bleaching interferes with pronase-mediated chorion removal (Mandrell et al. 2012).

A protocol using buffered chlorine would be more precise, but this is not practical with large-scale and frequent treatments as used in most zebrafish laboratories. Whereas the germicidal capability of chlorine is profoundly affected by $\mathrm{pH}$, we realize that buffering chlorine for egg treatments is not employed in zebrafish laboratories. Considering that water hardness and alkalinity directly influence the buffering capacity of water, chlorine at a given concentration will have different germicidal and toxic effects between laboratories. Hence, $\mathrm{pH}$ of egg-disinfectant media should be monitored, at least periodically.

The recommended dosages we provide here will not be $100 \%$ effective for the spores of the microsporidium Pseudoloma neurophilia, the most common zebrafish pathogen in research laboratories. However, increasing the concentration from 50 to $100 \mathrm{ppm}$ unbuffered chlorine solution increased killing of $P$. neurophilia spores from about 61 to $82 \%$ (Ferguson et al. 2007). These levels would likely be effective for a marked reduction of bacterial pathogens associated with zebrafish. For example, $50 \mathrm{ppm}$ chlorine is very effective against Aeromonas species. (Goñi-Urriza et al. 2000). This is particularly important as the highly pathogenic Gram-negative bacterium Edwardsiella ictaluri has recently been detected in zebrafish held in quarantine at several zebrafish laboratories (Hawke et al. 2013). Mycobacteriosis is also very common in zebrafish facilities caused most often by Mycobacterium chelonae, $M$. marinum, and M. haemophilum (Whipps et al. 2012). Mycobacteria are generally more resistant to chlorine than Gram-negative bacteria, and within the genus, there is great variability. For example, fastgrowing environmental species (e.g. M. chelonae and $M$. fortuitum) are more resistant than M. marinum (Bardouniotis et al. 2003, Mainous \& Smith 2005). Studies are underway (C. Whipp pers. comm.) evaluating the germicidal properties of chlorine using $M$. chelonae and $M$. marinum isolates from zebrafish, including our recommended protocol of 5 or 10 min unbuffered chlorine.

It should be noted that all of our experiments were conducted with the 5D line of zebrafish, a very robust outbred line derived from pet store fish and used extensively for toxicology studies at the SARL. Variations in quality and viability of eggs and embryos has been documented in several fish species in aquaculture, including zebrafish (Lawrence 2007) and has been attributed to several factors, including genetics, contaminants in the water and parents, nutritional and disease status of parents, and variations in temperature (Brooks et al. 1997, Schreck et al. 2001, Bobe \& Labbé 2010). The zebrafish research community uses a wide variety of fish lines (Trevarrow \& Robison 2004). Certain inbred lines are less fit than others (Monson \& Sadler 2010, Meyer et al. 2013), whereas we are not aware of documentation of variability in chlorine toxicity of embryos amongst different lines; thus caution should be exercised when applying the treatment protocols that we have recommend here to other, perhaps more fragile populations.

Given the instability of chlorine, alternative disinfectants should be investigated. For example, iodine is routinely used in food fish aquaculture to disinfect eggs, and a few zebrafish laboratories are now using this disinfectant to treat eggs (Milligan-Myhre et al. 2011). Regardless of the efficacy of disinfectants in destroying microorganisms, these will not prevent the transmission of intraovum pathogens, such as Pseudoloma neurophilia (Sanders \& Kent 2013).

Acknowledgements. This study was supported by grants from the National Institutes of Health NIH NCRR 5R24RR017386-02, P30ES000210, and RC4ES019764.

\section{LITERATURE CITED}

Bardouniotis E, Ceri H, Olson ME (2003) Biofilm formation and biocide susceptibility testing of Mycobacterium fortuitum and Mycobacterium marinum. Curr Microbiol 46: 28-32

Bobe J, Labbé C (2010) Egg and sperm quality in fish. Gen Comp Endocrinol 165:535-548

> Brooks S, Tyler CR, Sumpter JP (1997) Egg quality in fish: What makes a good egg? Rev Fish Biol Fish 7: 387-416

Clark RM, Eleanor RJ, Hoff J (1989) Analysis of inactivation of Giardia lamblia by chlorine. J Environ Eng 115:80-90 
Ferguson JA, Watral V, Schwindt AR, Kent ML (2007) Spores of two fish microsporidia (Pseudoloma neurophilia and Glugea anomola) are highly resistant to chlorine. Dis Aquat Org 76:205-214

Goñi-Urriza M, Pineau L, Capdepuy M, Roques C, Caumette P, Quentin C (2000) Antimicrobial resistance of mesophilic Aeromonas spp. isolated from two European rivers. J Antimicrob Chemother 46:297-301

Harper C, Lawrence C (2011) The laboratory zebrafish. CRC Press, Boca Raton, FL

Hawke JP, Kent ML, Rogge M, Baumgartner W and others (2013) Edwardsiellosis caused by Edwardsiella ictaluri in laboratory populations of zebrafish Danio rerio. J Aquat Anim Health 25:171-183

Health Canada (2004) Guidelines for Canadian drinking water quality: supporting documentation-Protozoa: Giardia and Cryptosporidium. Water Quality and Health Bureau, Healthy Environments and Consumer Safety Branch, Health Canada, Ottawa

> Kim KT, Tanguay RL (2013) Integrating zebrafish toxicology and nanoscience for safer product development. Green Chem 15:872-880

Lawrence C (2007) The husbandry of zebrafish (Danio rerio): a review. Aquaculture 269:1-20

Mainous ME, Smith SA (2005) Efficacy of common disinfectants against Mycobacterium marinum. J Aquat Anim Health 17:284-288

- Mandrell D, Truong L, Jephson C, Sarker MR and others (2012) Automated zebrafish chorion removal and single embryo placement: optimizing throughput of zebrafish developmental toxicity screens. J Lab Autom 17:66-74

Meyer BM, Froehlich JM, Galt NJ, Biga PR (2013) Inbred strains of zebrafish exhibit variation in growth performance and myostatin expression following fasting. Comp

Editorial responsibility: Thomas Braunbeck, Heidelberg, Germany
Biochem Physiol A Mol Integr Physiol 164:1-9

> Milligan-Myhre K, Charette JR, Phennicie RT, Stephens WZ, Rawls JF, Guillemin K, Kim CH (2011) Study of host-microbe interactions in zebrafish. Methods Cell Biol 105:87-116

Monson CA, Sadler KC (2010) Inbreeding depression and outbreeding depression are evident in wild-type zebrafish lines. Zebrafish 7:189-197

Morgan RP, Prince RD (1997) Chlorine toxicity to eggs and larvae of five Chesapeake Bay fishes. Trans Am Fish Soc 106:380-385

Sanders JL, Kent ML (2013) Verification of intraovum transmission of a microsporidium of vertebrates: Pseudoloma neurophilia infecting the zebrafish, Danio rerio. PLoS ONE 8:e76064

Schreck CB, Contreras-Sanchez W, Fitzpatrick MS (2001) Effects of stress on fish reproduction, gamete quality, and progeny. Aquaculture 19:3-24

- Trevarrow B, Robison B (2004) Genetic backgrounds, standard lines, and husbandry of zebrafish. Methods Cell Biol 77:599-616

> Truong L, Harper SL, Tanguay RL (2011) Evaluation of embryotoxicity using the zebrafish model. Methods Mol Biol 691:271-279

> Vaerewijck MJM, Sabbe K, Baré J, Spengler HP, Favoreel HW, Houf K (2012) Assessment of the efficacy of benzalkonium chloride and sodium hypochlorite against Acanthamoeba polyphaga and Tetrahymena spp. J Food Prot 75:541-546

Westerfield M (2007) The zebrafish book: a guide for the laboratory use of zebrafish (Danio rerio), 5th edn. University of Oregon Press, Eugene, OR

- Whipps CM, Lieggi C, Wagner R (2012) Mycobacteriosis in zebrafish colonies. ILAR J 53:95-105

Submitted: July 30, 2013; Accepted: October 10, 2013 Proofs received from author(s): December 18, 2013 